\title{
Efficacy of Glucosamine Sulphate in Skin Ageing: Results from an ex vivo Anti-Ageing Model and a Clinical Trial
}

\author{
Audrey Gueniche Isabelle Castiel-Higounenc \\ L'Oréal Research and Innovation, Aulnay-sous-Bois, France
}

\author{
Keywords \\ Collagen · Glucosamine sulphate - Skin ageing · \\ Skin structure · Skin physiology
}

\begin{abstract}
Background: Glucosamine sulphate (GS) is essential in the biosynthesis of glycolipids, glycoproteins, glycosaminoglycans (GAGs), hyaluronate, and proteoglycans. Connective tissues primarily contain collagen and proteoglycans and play an important role in skin ageing. Objective: The objectives were to assess ex vivo the impact of GS on skin ageing parameters and in vivo the effect of GS on the skin physiology of mature healthy volunteers after oral intake. Methods: The impact of GS on skin ageing was assessed ex vivo via different immunohistochemical assays and histology and via a clinical study using biopsies. Modulation of selected skin physiology markers was assessed by real-time quantitative PCR on skin punch biopsies obtained from 8 healthy $>50$-year-old women having ingested GS $250 \mathrm{mg}$ once daily for 8 weeks. Results: Ex vivo, GS significantly (all $p \leq 0.02$ ) increased the expression of CD44 and collagen type IV, the epidermis GAG level, and collagen type I synthesis. After 8 weeks of oral GS administration, a significantly increased expression was observed at the mRNA level for vimentin, fibromodulin, biglycan, xylosyl transferase, hyaluronan synthase, collagen types I and III, bone morphogenic protein-1, and
\end{abstract}

decorin (all $p \leq 0.05$ ). Conclusion: Both experiments showed that GS has a positive effect on epidermal and dermal markers associated with age.

(c) 2017 S. Karger AG, Basel

\section{Introduction}

All body tissues undergo intrinsic, or natural, ageing, which is most apparent on the skin when fine wrinkles become visible [1]. These wrinkles are caused by atrophy of the dermis due to loss of collagen and hydration and degeneration of the elastic fibre network $[2,3]$.

Besides this natural ageing process, the exposed skin is also subject to extrinsic ageing caused by environmental factors such as UV rays [4]. At a histological level, such skin shows general atrophy of the extracellular matrix with reduced elastin and disintegration of elastic fibres [5]. In comparison with intrinsically aged skin, photoaged skin appears coarse, roughened, and deeply wrinkled with marked loss of elasticity and recoil $[6,7]$. This is caused by the loss of the fibrillin-rich microfibrillar architecture in the papillary dermis. At a microscopic level, extrinsically aged skin contains an important accumulation of dystrophic elastotic material in the reticular dermis [8]. 
Glucosamine (GS) is an aminomonosaccharide that is naturally present in all human tissues. It is produced in the body by the addition of an amino group to glucose which is then acetylated to $\mathrm{N}$-acetylglucosamine [9]. While $\mathrm{N}$ acetylglucosamine is attached to glycoproteins located on the extracellular side of the cell membrane, playing an important role in intercellular recognition [10], GS is the primary amino sugar substrate for the biosynthesis of hyaluronic acid and of heparan sulphate, and subsequently for the production of proteoglycans [9]. All of those are beneficial for skin hydration, wrinkle reduction, and management of skin pigmentation disorders [9, 11-13].

The objectives of the studies reported hereafter were to assess ex vivo, by using immunohistochemical assays and histological analyses, the impact of GS on skin ageing parameters and the effect of GS on the skin physiology of mature healthy volunteers after oral intake.

\section{Methods}

Methods: Ex vivo Study

Sample Preparation

Normal human skin samples were obtained from 3 anonymous Caucasian healthy women aged between 32 and 50 years. Surgical residue was harvested according to French regulation and procurement of written informed consent from the patient. Skin samples, 2 from breast reduction surgery and 1 from abdominal plastic surgery protected from UV radiation, were used [14]. The surgical residues were washed 3 times with antibiotics and then cut into $1-\mathrm{cm}^{2}$ full-thickness pieces. Subcutaneous fat and lower dermis were mechanically removed under a stereomicroscope using a surgical scalpel. Skin biopsies were placed on culture inserts (filter pore size $12 \mu \mathrm{m}$; Costar, Poly-Labo Paul Block, France) with the epithelium uppermost at an air/liquid interface. The inserts were set on 12-well plates (Costar), and culture medium was added to the wells so that the surface of the medium reached the filter level. Organ cultures were performed using Dulbecco's minimal essential medium (Gibco BRL) containing antibiotics $(100 \mathrm{U} / \mathrm{mL}$ penicillin and $100 \mu \mathrm{g} / \mathrm{mL}$ streptomycin; Gibco BRL, USA), $200 \mu \mathrm{g} / \mathrm{mL}$ L-glutamine (Gibco BRL), bovine pituitary extract, growth factors and foetal calf serum (DAP, France). Cohesion between skin and insert was obtained with a polysiloxane vinyl seal in such a way that neither skin retraction nor lateral passage of any applied topical product towards the dermis was possible. Skin samples were kept under these survival conditions for $5 \mathrm{~h}$ at $37^{\circ} \mathrm{C}$ in a humidified incubator with $5 \% \mathrm{CO}_{2}$.

\section{Exposure to GS}

Three samples from each donor were prepared:

- unexposed skin: no added GS to culture medium;

- skin + GS at $150 \mu \mathrm{g} / \mathrm{mL}$;

- skin + GS at $300 \mu \mathrm{g} / \mathrm{mL}$.

GS was distributed via the culture medium.

Cultures were stopped after 7 days of incubation, and skin samples were frozen at $-20^{\circ} \mathrm{C}$ or fixed in formalin $4 \%$ for immunohistochemical assays.

Oral Glucosamine Sulphate in Skin

Ageing
Samples of 6- $\mu \mathrm{m}$ thickness were prepared using cryosectioning and mounted on cleaned glass slides. After air-drying for $15 \mathrm{~min}$, the sections were fixed in ice-cold acetone for $10 \mathrm{~min}$. The sections were air-dried again for 2-24 h. Covered in aluminium foil, sections were stored at $-20^{\circ} \mathrm{C}$.

One skin sample from each donor was frozen after 14 days of incubation allowing for the measuring of collagen concentrations.

Immunohistochemical Assays

The following assays were performed according to the providers' standard protocols of use.

Collagen Type IV. Collagen IV was detected using the mouse monoclonal antibody PHM-12 (diluted 1:50, AbCys, Les Ulis, France). A double-layer immunoperoxidase test (ABC kit, Vector Laboratories, Burlingame, CA, USA) was used. 3-Amino-9-ethylcarbazole was used for staining. The intensity of immunohistochemical marking of the granulous layer (filaggrin) and for the dermo-epidermal junction (collagen type IV) was assessed using a semiquantitative scoring system from $0=$ no marking to $4=$ very important intensity.

CD44 (Receptor of Hyaluronic Acid). Immunodetection of CD44 was performed using monoclonal antibodies (diluted 1:20, NCL-CD44-2, Novacastra, Buffalo Grows, IL, USA). The amplification required the CSA kit (DAKO, Carpinteria, CA, USA), 3-amino-9-ethylcarbazole for staining. The expression of CD44 was assessed on a semiquantitative scoring system from $0=$ low to $4=$ very important intensity.

Collagen Type I. Collagen type I was detected in the dermis by using the mouse anticollagen antibody type I human clone 4F6 (diluted 1:300, Southern Biotech, Birmingham, AL, USA), and shown by using the fluorescent antibody goat immunoglobulin IgG2b. The intensity of staining in the superficial dermal layer below the dermal-epidermal junction was assessed on a semiquantitative scoring system ranging from $0=$ low to $4=$ very important intensity.

Histological Analysis of Glycosaminoglycans. Glycosaminoglycans (GAGs) were evidenced by using the Hale stain method (Alcian blue, Sigma Aldrich, Cergy-Pontoise, France). The histological analysis allowed for showing potential changes in the amount of GAGs in the epidermis and the superficial to deep layers of the dermis, using a semiquantitative scoring system ranging from $0=$ no intensity to $4=$ very important intensity.

Measuring of Total Collagen. After 14 days of incubation, skin samples were lysed overnight at $4^{\circ} \mathrm{C}$ in a solution containing acetic acid at $0.5 \mathrm{M}$ and pepsin. This method allowed the newly synthesized collagen (neocollagen) to being harvested [15]. After crushing in a potter, the amount of collagen (in micrograms per milligram) was determined by using spectrometry at $540 \mathrm{~nm}$. The acidsoluble collagen was detected after staining with the marker Sirius red (Sircol Collagen Assay, Interchim Montluçon, France) [16]. Final results were expressed in micrograms of collagen per milligram of fresh weight.

The list of skin biomarkers tested ex vivo is provided in Table 1.

\section{Statistics}

Statistical analyses were performed by using the paired difference test (Student test) and considering a risk alpha of 5\% versus the unexposed skin samples. All statistical analyses were carried out using SAS Enterprise Guide version 4.2 (SAS Institute, Cary, NC, USA) and SPSS version 17 (IBM, Armonk, NY, USA) statistical software. 
Methods: Healthy Volunteer Study

Ethical Considerations

This monocentric study was conducted in accordance with the Good Clinical Practices and the principles of the Declaration of Helsinki. An independent ethics committee of Paris-Broussais, France, provided approval prior to the start of the study, and all participating subjects provided written informed consent prior to inclusion.

Population Studied

Eight healthy females with phototypes II-III according to the Fitzpatrick phototype scale [17] were recruited. The 2 main factors that influence skin type are: genetic disposition and reaction to sun exposure with tanning habits; type II usually burns and tans minimally (white; fair; blond or red hair; blue, green, or hazel eyes); type III sometimes shows mild burn but tans uniformly (cream white; fair with any hair or eye colour). Healthy females at least 50 years of age, with menopause started at least 2 years prior to the start of the study and exempt of any hormonal substitutive therapy, were selected for this study.

Product Tested

All participants were supplemented once daily for 8 weeks with 1 hard capsule containing 250 mg of GS (INNEOV, Asnières-surSeine, France).

\section{Sample Preparation}

Two 3-mm punch biopsies were collected from the subjects' inner side of the forearm before the supplement intake (week 0). Dermis and hypodermis were collected separately. Two punch biopsies were also collected after 8 weeks (week 8) on the same forearm, $2 \mathrm{~cm}$ away from the initial biopsies.

Preparation of RNA for Real-Time Quantitative PCR

Analyses and Quality Assessment

Skin biopsies were crushed in liquid nitrogen (Mikro Dismembrator S, B. Braun Biotech International, Berlin, Germany) to extract and purify total RNA by using the Ambion Ribo Pure kit (Life Technologies, Austin, TX, USA). The amount of RNA collected was determined by measuring the optical density at $260 \mathrm{~nm}$ (Nanodrop) and the quality of the RNA by the ratio of optical densities at $260 / 280 \mathrm{~nm}$. The integrity of the purified RNA was further analysed in the BioAnalyzer Agilent (Agilent Technologies, Santa Clara, CA, USA). Solutions of RNA were brought to a theoretical concentration of $4 \mathrm{ng} / \mu \mathrm{L}$.

Each purified RNA sample, except 1 that was degraded at week 8 , met the required quality criteria.

Determination of Biomarkers

The concentration of RNA was determined by measuring the ribosomal RNA (28S rRNA) and specific gene transcripts by realtime PCR quantitatively through adding in each tube an internal standard made of a synthetic RNA cotranscribed and co-amplified with the cellular RNA as described by Nusgens et al. [18]. Week 0 and week 8 samples from each participant were analysed in the same real-time PCR run and on the same sodium dodecylsulphate slab gel polyacrylamide electrophoresis. Results were expressed in arbitrary units per unit of $28 \mathrm{~S}$ rRNA.

The effect of GS supplementation on the dermis was evaluated by measuring the mRNA for the mesenchymal marker vimentin,
Table 1. Skin biomarkers and macromolecules tested ex vivo and in vivo

Skin biomarkers assayed ex vivo

CD44

Collagen type IV

Glycosaminoglycan

Total neocollagen

Human skin macromolecules assessed in vivo

Keratin-10

Vimentin

Glyceraldehyde 3-phosphate dehydrogenase

Glyceraldehyde phosphate dehydrogenase

Collagen type I

Collagen type III

Bone morphogenic protein-1

Decorin

Lumican

Fibromodulin

Biglycan

Xylosyl transferase

Hyaluron synthase

Actin

an intermediary filament of the cytoskeleton of connective tissue cells such as fibroblasts [19]. The effect on the hypodermis was evaluated by glycerol 3-phosphate dehydrogenase, an enzyme more specifically expressed by adipocytes [20]. The overall metabolic activity of the cells was determined by the level of mRNA for the mitochondrial enzyme glyceraldehyde phosphodehydrogenase.

A complete list of skin macromolecules tested in vivo is provided in Table 1.

\section{Statistical Analyses}

For each participant, the mean mRNA expression in the 2 samples at week 8 was compared to the mean value at week 0 with a ratio week 8 :week $0=1.00$ of the 2 values, meaning no change. The statistical significance of the mean ratio week 8:week 0 in the study group was calculated by using the Student test for paired samples. All statistical analyses were carried out using SAS Enterprise Guide version 4.2 (SAS Institute, Cary, NC, USA) and SPSS version 17 (IBM, Armonk, NY, USA) statistical software.

\section{Results}

\section{Results: Ex vivo Study}

\section{CD44 Expression}

A statistically significant increase in the mean score of CD44 expression in the epidermis was observed after exposure to GS at 150 and $300 \mu \mathrm{g} / \mathrm{mL}(3.88 \pm 0.18, p=0.008$, and $3.96 \pm 0.07, p=0.02$, respectively) corresponding to a moderate percent increase (5.7 and $8.0 \%$, respectively) as compared to the unexposed sample.
Gueniche/Castiel-Higounenc 
Table 2. Mean $( \pm$ SD) mRNA expression levels at week 0 and individual mean $( \pm S D)$ ratios at week 8 and week 0

\begin{tabular}{lrl}
\hline & $\begin{array}{l}\text { Week 0 } \\
\text { GS 250 mg } \\
(n=8)\end{array}$ & $\begin{array}{l}\text { Week 8/week 0 ratio } \\
\text { GS 250 mg } \\
(n=8)\end{array}$ \\
\hline Keratin-10 & $10.51 \pm 1.54$ & $1.06 \pm 0.17$ \\
Vimentin & $7.63 \pm 1.95$ & $1.28 \pm 0.33^{*}$ \\
G3PDH & $4.47 \pm 0.70$ & $1.08 \pm 0.19$ \\
GAPDH & $1.73 \pm 0.33$ & $1.03 \pm 0.24$ \\
Collagen type I & $1.68 \pm 0.71$ & $1.72 \pm 0.69^{* *}$ \\
Collagen type III & $1.03 \pm 0.35$ & $1.76 \pm 0.68^{* *}$ \\
BMP1 & $2.30 \pm 0.36$ & $1.32 \pm 0.31^{* *}$ \\
Decorin & $15.33 \pm 3.27$ & $1.11 \pm 0.11^{* *}$ \\
Lumican & $14.99 \pm 2.93$ & $1.12 \pm 0.18$ \\
Fibromodulin & $13.64 \pm 2.82$ & $1.10 \pm 0.12^{*}$ \\
Biglycan & $10.87 \pm 2.83$ & $1.15 \pm 0.19^{*}$ \\
Xylosyl transferase & $6.42 \pm 2.40$ & $1.26 \pm 0.33^{*}$ \\
Hyaluronan synthase & $4.00 \pm 1.42$ & $1.62 \pm 1.42^{*}$ \\
Actin & $10.35 \pm 1.82$ & $1.12 \pm 0.19$ \\
\hline
\end{tabular}

G3PDH, glyceraldehyde 3-phosphate dehydrogenase; GAPDH, glyceraldehyde phosphate dehydrogenase; BMP1, bone morphogenic protein-1. $* p \leq 0.05,{ }^{* *} p \leq 0.01$.

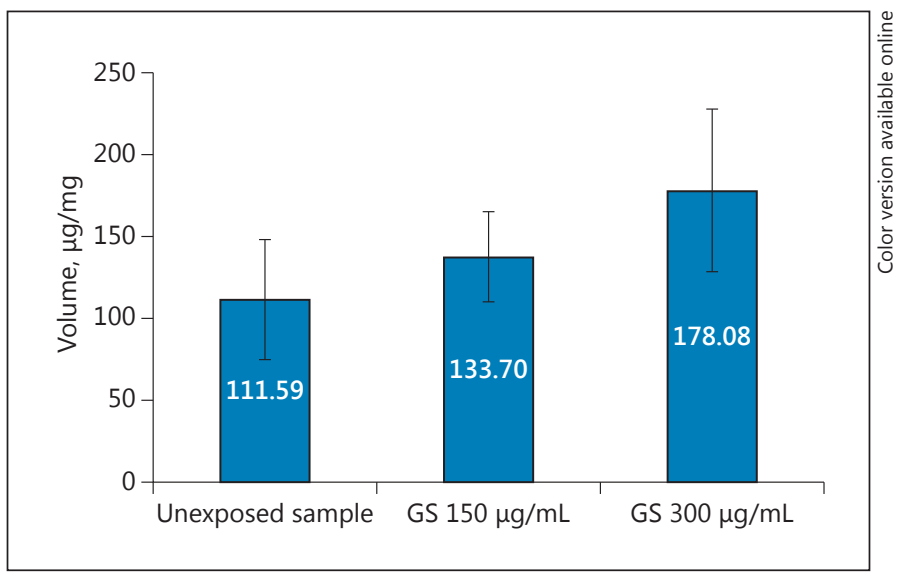

Fig. 1. Synthesis of neocollagen. A significant increase in neocollagen was observed (both $p \leq 0.047$ ) in the samples incubated in medium with added GS 150 or $300 \mu \mathrm{g} / \mathrm{mL}$ as compared to without (unexposed sample). The difference between both GS doses was significant $(p<0.05)$ in favour of GS $300 \mu \mathrm{g} / \mathrm{mL}$. GS, glucosamine sulphate.

\section{Collagen Type IV}

A statistically significant increase in collagen type IV expression with a mean score of $2.32 \pm 0.24$ compared to the unexposed skin sample $(1.64 \pm 0.88, p=0.014)$ was observed with GS $300 \mu \mathrm{g} / \mathrm{mL}$. The increase was not significant for GS $150 \mu \mathrm{g} / \mathrm{mL}(2.07 \pm 0.31)$. The difference between both doses was statistically significant $(p=0.04)$.

\section{Glycosaminoglycans}

A statistically significant increase in GAGs was observed in the epidermis of the ex vivo samples from all 3 donors after exposure to GS 150 and $300 \mu \mathrm{g} / \mathrm{mL}$, with mean scores of $2.35 \pm 0.34$ and $2.42 \pm 1.27$, respectively, as compared to the unexposed sample $(1.15 \pm 0.88)$, both $p \leq 0.015$.

\section{Total Collagen}

A dose-dependent effect on the synthesis of neocollagen was observed. The amount of neocollagen significantly increased in samples from all donors up to 133.70 \pm 27.3 and $178.08 \pm 50.25 \mu \mathrm{g} / \mathrm{mg}$ after exposure to GS 150 and $300 \mu \mathrm{g} / \mathrm{mg}$, respectively, compared to the unexposed sample $(111.59 \pm 36.19 \mu \mathrm{g} / \mathrm{mg}$ ) (Fig. 1). The difference was statistically significant for both GS concentrations $(p=0.047)$.

\section{Results: Healthy Volunteer Study}

\section{Volunteer Population}

All 8 volunteers met the inclusion criteria, received GS, and were biopsied.

Individual Ratios between the mRNA Level at Week 8 and Week 0

The ratio of expression of mRNA for vimentin, fibromodulin, biglycan, xylosyl transferase, hyaluronan synthase, collagen type I and III, the bone morphogenic protein-1 (BMP1), and decorin significantly increased from week 0 to week 8 (all $p \leq 0.05$ ). The increase was the most important for collagen type I and III, BMP1, and decorin. There was no change for the other biomarkers investigated in the volunteers following GS supplementation.

Detailed results after 8 weeks of daily intake are provided in Table 2.

\section{Discussion and Conclusion}

The present ex vivo and in vivo studies aimed at assessing the impact of GS throughout selected markers of the skin ageing process and the skin physiology in healthy female volunteers, and confirm that not only the derivate $\mathrm{N}$-acetylglucosamine, as reported by Bissett [9], but also the mother compound GS has an impact on the expres- 
sion of biomarkers of skin ageing, as shown ex vivo and in vivo.

Indeed, we demonstrated that GS significantly increased ex vivo the expression of the hyaluronic acid receptor CD44 in the epidermis and that of collagen type IV. GS stimulated the synthesis of neocollagen in a dosedependent way with samples incubated in a medium containing GS at $300 \mu \mathrm{g} / \mathrm{mL}$ presenting with the highest amount of neocollagen.

It is commonly accepted that CD44 plays an important role in maintaining matrix stability and regulating cellcell adhesion and that it is the principal cell surface receptor of hyaluronan $[21,22]$. Therefore, increasing the expression of CD44 is important in the management of skin ageing: the significant increase in GAGs enhances the improved water-holding capacities and maintenance of water content of tissues, thus supporting this observation $[23,24]$. The fact that GS increased the expression of mRNA for collagen type IV is particularly important as this RNA expression is central to cell adhesion, migration, differentiation, and growth, which is another important issue in body tissue ageing [25].

Our in vivo study in healthy female volunteers showed that an 8-week oral supplementation with GS at $250 \mathrm{mg}$ resulted in a significant increase in mRNA expression for vimentin, a type III intermediate filament protein. Like collagen, vimentin plays an important role in cell adhesion, migration, and differentiation, which is an issue in tissue regeneration [26, 27]. Furthermore, RNA expression was increased for fibromodulin, an important element in the wound-healing process [28]; biglycan, involved in structural, space-filling functions and many other physiological regulations in the skin; xylosyl transferase, playing an important role in the synthesis of proteoglycans especially heparan sulphate [29]; hyaluronan synthase, responsible for the synthesis of hyaluronan, a major extracellular matrix component in the epidermis and essential for skin repair [30]; decorin, to which binding results in an assembly of collagen fibrils and in the inhibition of cleavage of collagen fibrils by matrix metalloprotease-1 [31]; and BMP1, involved in the molecular network regulating homeostasis in the skin [32].

Healthy female volunteers showed that an 8-week oral supplementation with GS at $250 \mathrm{mg}$ resulted in a significant increase in mRNA expression of collagen I and III very important for skin tension, elasticity, and healing [33].

Conversely, we could not demonstrate in vivo the impact of GS on the mRNA of a certain number of other markers involved in skin repair and wound healing as well as in limiting inflammation, such as the phospholipid-binding proteins, keratin-10, glycerol 3-phosphate dehydrogenase, glyceraldehyde phosphodehydrogenase, lumican, and actin. All of them have been described in the literature as playing a role in wound healing [19, 34-36]. These negative study outcomes may be related to study conditions, and further research may be needed to confirm or contradict our findings.

Nevertheless, results from our studies demonstrated that GS improves ex vivo the expression of the hyaluronic acid receptor CD44 in the epidermis, the epidermal glycosaminoglycan content and de novo collagen synthesis. Furthermore, our ex vivo results confirm the rationale for further investigations of GS as a skin anti-ageing ingredient.

The present primary data were strengthened by the outcome obtained in vivo following oral intake of GS, resulting in an improvement of various age-related markers involved in epidermal differentiation or extracellular dermal matrix formation.

\section{Acknowledgements}

The studies were funded by L'Oréal France and Nestlé Switzerland. The authors acknowledge the formulation support of Marjory Graffin, INNEOV France and the writing support of Karl Patrick Goritz, SMWS - Scientific and Medical Writing Services, France.

\section{Statement of Ethics}

This monocentric study was conducted in accordance with Good Clinical Practices and the principles of the Declaration of Helsinki. An independent ethics committee of Paris-Broussais, France, provided approval prior to the start of the study, and all participating subjects provided written informed consent prior to inclusion.

\section{Disclosure Statement}

All authors are employees of L’Oréal.

References

1 Watson RE, et al: Effects of a cosmetic "antiageing" product improves photoaged skin. $\mathrm{Br}$ J Dermatol 2009;161:419-426.

2 Uitto J: The role of elastin and collagen in cutaneous aging: intrinsic aging versus photoexposure. J Drugs Dermatol 2008;7:s12-s16.

3 Chung JH, et al: Modulation of skin collagen metabolism in aged and photoaged human skin in vivo. J Invest Dermatol 2001;117: $1218-1224$.
40

Skin Pharmacol Physiol 2017;30:36-41 DOI: $10.1159 / 000450832$
Gueniche/Castiel-Higounenc 
4 Fisher GJ, et al: Mechanisms of photoaging and chronological skin aging. Arch Dermatol 2002;138:1462-1470.

5 Braverman IM, Fonferko E: Studies in cutaneous aging. I. The elastic fiber network. J Invest Dermatol 1982;78:434-443.

6 Smith JG Jr, Davidson EA, Sams WM Jr, Clark RD: Alterations in human dermal connective tissue with age and chronic sun damage. J Invest Dermatol 1962;39:347-350.

7 Warren R, et al: Age, sunlight, and facial skin: a histologic and quantitative study. J Am Acad Dermatol 1991;25:751-760.

8 Watson RE, Griffiths CE, Craven NM, Shuttleworth CA, Kielty CM: Fibrillin-rich microfibrils are reduced in photoaged skin. Distribution at the dermal-epidermal junction. J Invest Dermatol 1999;112:782-787.

9 Bissett DL: Glucosamine: an ingredient with skin and other benefits. J Cosmet Dermatol 2006;5:309-315.

10 Stryer L: Biochemistry. New York, Freeman \& Co, 1988, pp 284-310.

11 Weindl G, Schaller M, Schäfer-Korting M, Korting HC: Hyaluronic acid in the treatment and prevention of skin diseases: molecular biological, pharmaceutical and clinical aspects. Skin Pharmacol Physiol 2004;17:207-213.

12 Sayo T, Sakai S, Inoue S: Synergistic effect of $\mathrm{N}$-acetylglucosamine and retinoids on hyaluronan production in human keratinocytes. Skin Pharmacol Physiol 2004;17:77-83.

13 Imokawa G, Mishima Y: Analysis of tyrosinases as asparagin-linked oligosaccharides by concanavalin A lectin chromatography: appearance of new segment of tyrosinases in melanoma cells following interrupted melanogenesis induced by glycosylation inhibitors. J Invest Dermatol 1985;85:165-168.

14 Freshney R, Freshney M: Culture of Epithelial Cells, ed 2. New York, Wiley-Liss, 2002, pp 109-120.
15 Colombatti A, Bonaldo P, Ainger K, Bressan GM, Volpin D: Biosynthesis of chick type VI collagen. I. Intracellular assembly and molecular structure. J Biol Chem 1987;262:1445414460.

16 Chen CZ, Raghunath M: Focus on collagen: in vitro systems to study fibrogenesis and antifibrosis state of the art. Fibrogenesis Tissue Repair 2009;2:7.

17 Fitzpatrick TB: The validity and practicality of sun-reactive skin types I through VI. Arch Dermatol 1988;124:869-871.

18 Nusgens BV, et al: Topically applied vitamin C enhances the mRNA level of collagens I and III, their processing enzymes and tissue inhibitor of matrix metalloproteinase 1 in the human dermis. J Invest Dermatol 2001;116: 853-859.

19 Goodpaster T, et al: An immunohistochemical method for identifying fibroblasts in formalin-fixed, paraffin-embedded tissue. J Histochem Cytochem 2008;56:347-358.

20 Cook JR, Kozak LP: SN-glycerol-3-phosphate dehydrogenase gene expression during mouse adipocyte development in vivo. Dev Biol 1982;92:440-448.

21 Simpson RM, et al: Age-related changes in pericellular hyaluronan organization leads to impaired dermal fibroblast to myofibroblast differentiation. Am J Pathol 2009;175:19151928.

22 Dos Santos M, et al: In vitro 3-D model based on extending time of culture for studying chronological epidermis aging. Matrix Biol 2015;47:85-97.

23 Taylor KR, Gallo RL: Glycosaminoglycans and their proteoglycans: host-associated molecular patterns for initiation and modulation of inflammation. FASEB J 2006;20:9-22.

24 Jung JY, et al: Acute UV irradiation increases heparan sulfate proteoglycan levels in human skin. J Korean Med Sci 2012;27:300-306.

25 Aumailley M, Timpl R: Attachment of cells to basement membrane collagen type IV. J Cell Biol 1986;103:1569-1575.
26 Ivaska J, Pallari HM, Nevo J, Eriksson JE: Novel functions of vimentin in cell adhesion, migration, and signaling. Exp Cell Res 2007; 313:2050-2062.

27 Dave JM, Bayless KJ: Vimentin as an integral regulator of cell adhesion and endothelial sprouting. Microcirculation 2014;21:333344 .

28 Zheng Z, et al: Fibromodulin enhances angiogenesis during cutaneous wound healing. Plast Reconstr Surg Glob Open 2014;2:e275.

29 Franco CR, et al: Heparan sulfate and control of cell division: adhesion and proliferation of mutant CHO-745 cells lacking xylosyl transferase. Braz J Med Biol Res 2001;34:971-975.

30 Tammi R, Pasonen-Seppänen S, Kolehmainen E, Tammi M: Hyaluronan synthase induction and hyaluronan accumulation in mouse epidermis following skin injury. J Invest Dermatol 2005;124:898-905.

31 Li Y, et al: Age-dependent alterations of decorin glycosaminoglycans in human skin. Sci Rep 2013;3:2422.

32 Botchkarev VA: Bone morphogenetic proteins and their antagonists in skin and hair follicle biology. J Invest Dermatol 2003;120: 36-47.

33 Cheng W: The content and ratio of type I and III collagen in skin differ with age and injury. Afr J Biotechnol 2011;10:2524-2529.

34 Liu XJ, et al: Lumican accelerates wound healing by enhancing a $2 \beta 1$ integrin-mediated fibroblast contractility. PLoS One 2013;8: e67124.

35 Rivera-Gonzalez G, Shook B, Horsley V: Adipocytes in skin health and disease. Cold Spring Harb Perspect Med 2014;4:a015271.

36 Jensen JM, Schütze S, Neumann C, Proksch E: Impaired cutaneous permeability barrier function, skin hydration, and sphingomyelinase activity in keratin 10 deficient mice. J Invest Dermatol 2000;115:708-713. 